\title{
QUALITATIVE THEORY OF PARTIAL DIFFERENCE EQUATIONS (II): OSCILLATION CRITERIA FOR DIRECT CONTROL SYSTEMS IN SEVERAL VARIABLES
}

\author{
SUI SUN CHENG, SHENG-LI XIE AND BING-GEN ZHANG
}

\begin{abstract}
This paper is concerned with several direct control systems which are described by partial difference equations. By means of an averaging technique and several oscillation criteria for ordinary recurrence relations, we are able to establish several oscillation criteria for these control systems.
\end{abstract}

1. Discrete time control systems have been studied quite extensively. In contrast, direct control systems with several independent variables do not seem to have drawn as much attention. This is especially true in the particular area of oscillation of control systems described by partial difference equations.

In this paper, we consider several such systems and provide criteria for oscillations. A few preparatory terminologies will be needed. Let $u(i, j)$ be a real function of integral variables $i$ and $j$. We denote the partial differences of $u(i, j)$ by

$$
\begin{aligned}
\Delta_{1} u(i, j) & =u(i+1, j)-u(i, j) \\
\Delta_{2} u(i, j) & =u(i, j+1)-u(i, j) \\
\Delta_{1}^{2} u(i-1, j) & =\Delta_{1}\left(\Delta_{1} u(i-1, j)\right)=u(i+1, j)-2 u(i, j)+u(i-1, j) \\
\Delta_{2}^{2} u(i, j-1) & =\Delta_{2}\left(\Delta_{2} u(i, j-1)=u(i, j+1)-2 u(i, j)+u(i, j-1)\right.
\end{aligned}
$$

The usual $n$-th difference of a real function $v(i)$ of an integral variable $i$ is defined induction by

$$
\begin{aligned}
\Delta^{n} v(i) & =\Delta\left(\Delta^{n-1} v(i)\right), \quad n \geq 2, \\
\Delta v(i) & =v(i+1)-v(i) .
\end{aligned}
$$

Let $u(i, j)$ be a real function defined for $0 \leq i \leq n+1$ and $j \geq-\tau$ where $\tau$ is a nonnegative integer. If $u(i, j)>0$ for $1 \leq i \leq n$ and all large $j$, then $u(i, j)$ is said to be

Received March 23, 1994.

1991 Mathematics Subject Classification. 39A10, 35R10

Key words and phrases. Direct control systems, partial difference equations,oscillation. 
eventually positive. An eventually negative function $u(i, j)$ is similarly defined. If $u(i, j)$ is neither eventually positive nor eventually negative, we say that $u(i, j)$ is oscillatory [4].

To motivate our approach to discrete direct control systems, we first consider a prototype of the form

$$
\begin{aligned}
\Delta_{2} u(i, j)= & a_{0}(j) \Delta_{1}^{2} u(i-1, j)-q_{0}(j) u(i, j) \\
& -q_{1}(j) u(i, j-\sigma)+h(j) f(c(j) u(i, j)) \quad 1 \leq i \leq n, \quad j \geq 0
\end{aligned}
$$

where the delay $\sigma$ is a nonnegative integer, and $a_{0}(j), h(j), c(j)$ are nonnegative functions for $j \geq 0$. The control function is $h(j) f(c(j) u(i, j))$ and we shall assume that $f$ belongs to an angular domain $\Psi_{\theta}$ of the form

$$
\Psi_{\theta}=\{g \in C(-\infty, \infty) \mid g(0)=0, \quad 0 \leq g(\sigma) / \sigma \leq \theta \text { for } \sigma \neq 0\}
$$

For comparison, we mention that this system can be regarded as a discrete analog of the partial differential equation

$$
u_{t}(x, t)=a_{0}(t) u_{x x}(x, t)-q_{0}(t) u(x, t)-q_{1}(t) u(x, t-\sigma)+h(t) f(c(t) u(x, t)) .
$$

Under the additional conditions

$$
\begin{aligned}
& u(0, j)=0=u(n+1, j), \quad j \geq 0 \\
& u(i, j)=\varphi(i, j), \quad-\sigma \leq j \leq 0, \quad 0 \leq i \leq n+1
\end{aligned}
$$

where $\varphi$ is a given function satisfying $\varphi(0,0)=0=\varphi(n+1,0)$, it is not difficult to see that a solution $u(i, j ; \varphi)$ of (1) exists and is unique (this can be seen by writing (1) in the form

$$
u(i, j+1)=u(i, j)+F(i-1, i, i+1, j-\sigma, j)
$$

for $1 \leq i \leq n$ and $j \geq 0$ ).

Given an angular domain $\Psi_{\theta}$, suppose for any $f$ in $\Psi_{\theta}$, all solutions $u(i, j ; \varphi)$ of (1)-(3)-(4) are oscillatory, then we say that the control system (1) is $\Psi_{\theta}$-oscillatory. Otherwise, (1) is said to be $\Psi_{\theta}$-nonoscillatory.

We shall be concerned with conditions which are sufficient for oscillation of the system (1) (and other control systems to be specified later). For this purpose, we first mention the following result which can be verified in a straight forward manner (see [1] for a proof.)

Lemma 1. Let

$$
g(i, j)= \begin{cases}(n-i+1) j /(n+1) & 1 \leq j \leq i \\ (n-j+1) i /(n+1) & i \leq j \leq n\end{cases}
$$


Then $g(i, j)>0$ for $1 \leq i, j \leq n$ and

$$
\sum_{j=1}^{n} g(i, j) \Delta^{2} v(j-1)=-v(i)+\delta_{i}, \quad 1 \leq i \leq n
$$

where $\delta_{1}=n v(0) /(n+1), \delta_{n}=n v(n+1) /(n+1)$ and $\delta_{i}=0$ for $2 \leq i \leq n-1$.

Suppose now there is a function $f$ in $\Psi_{\theta}$ such that a solution $u(i, j)$ of $(1)$ satisfies $u(i, j)>0$ for $1 \leq i \leq n$ and $j \geq T$ or $u(i, j)<0$ for $1 \leq i \leq n$ and $j \geq T$. Let $\delta=\operatorname{sign} u(i, j)$ and $w(i, j)=\delta u(i, j)$. Then $w(i, j)>0$ for $1 \leq i \leq n$ and $j \geq T$, $w(i, j-\sigma)>0$ for $1 \leq i \leq n$ and $j \geq T+\sigma$. Multiplying (1) by $g(t, i)$ and summing the resulting equation, we obtain

$$
\begin{aligned}
\sum_{i=1}^{n} g(t, i) \Delta_{2} u(i, j)= & \sum_{i=1}^{n} g(t, i) a_{0}(j) \Delta_{1}^{2} u(i-1, j)-\sum_{i=1}^{n} g(t, i) q_{0}(j) u(i, j) \\
& -\sum_{i=1}^{n} g(t, i) q_{1}(j) u(i, j-\sigma)+\sum_{i=1}^{n} g(t, i) h(j) f(c(j) u(i, j)) .
\end{aligned}
$$

In view of Lemma 1 , condition (3) and the assumption on $f$, we have

$$
\begin{aligned}
& \Delta_{2} \sum_{i=1}^{n} g(t, i) w(i, j) \leq-a_{0}(j) w(t, j)-\sum_{i=1}^{n} g(t, i) q_{0}(j) w(i, j) \\
& -\sum_{i=1}^{n} g(t, i) q_{1}(j) w(i, j-\sigma)+\sum_{i=1}^{n} g(t, i) h(j) c(j) w(i, j) \theta,
\end{aligned}
$$

so that

$$
\begin{aligned}
\Delta_{2} \sum_{i=1}^{n} g(t, i) w(i, j) & \leq-\left[q_{0}(j)-h(j) c(j) \theta\right] \sum_{i=1}^{n} g(t, i) w(i, j) \\
& -q_{1}(j) \sum_{i=1}^{n} g(t, i) w(i, j-\sigma) .
\end{aligned}
$$

Summing the above inequality with respect to $t$, we obtain

$$
\begin{aligned}
\Delta_{2} \sum_{t, i=1}^{n} g(t, i) w(i, j) & \leq-\left[q_{0}(j)-h(j) c(j) \theta\right] \sum_{t, i=1}^{n} g(t, i) w(i, j) \\
& -q_{1}(j) \sum_{t, i=1}^{n} g(t, i) w(i, j-\theta) .
\end{aligned}
$$

This implies that

$$
\Delta v(j) \leq-\left[q_{0}(j)-h(j) c(j) \theta\right] v(j)-q_{1}(j) v(j-\sigma)
$$


for all large $j$, where

$$
v(j)=\sum_{t, i=1}^{n} g(t, i) w(i, j)
$$

which is eventually positive.

Let us denote $q_{0}(j)-h(j) c(j) \theta$ by $b_{\theta}(j)$. Then we can rewrite (8) as

$$
\left(1-b_{\theta}(j)\right) \Delta v(j) \leq-b_{\theta}(j) v(j+1)-q_{1}(j) v(j-\sigma)
$$

for all large j. Also, if we multiply both sides of $(8)$ by $\exp \left\{b_{\theta}(0)+\cdots+b_{\theta}(j)\right\}$, then it is not difficult to see that

$$
\begin{aligned}
\Delta\left\{v(j) \exp \sum_{i=0}^{j-1} b_{\theta}(i)\right\} & \leq\left\{1-\exp \left(-b_{\theta}(j)\right)-b_{\theta}(j)\right\} v(j) \exp \sum_{i=0}^{j} b_{\theta}(i) \\
& -q_{1}(j)\left\{\exp \sum_{i=j-\sigma}^{j} b_{\theta}(i)\right\} v(j-\sigma) \exp \sum_{i=0}^{j-\sigma-1} b_{\theta}(i)
\end{aligned}
$$

for all large $j$.

Thus assuming that $0 \leq b_{\theta}(j)<1$ for all large $j$, then it follows from (9) that

$$
\Delta v(j) \leq \frac{-q_{1}(j)}{1-b_{\theta}(j)} v(j-\sigma)
$$

for all large $j$. Note that the assumption that $1-b_{\theta}(j)>0$ for all large $j$ can be inferred from the assumption that $q_{1}(j)$ is eventually nonnegative. This is because (8) implies

$$
\left(1-b_{\theta}(j)\right) v(j) \geq v(j+1)+q_{1}(j) v(j-\sigma)>0
$$

for all large $j$.

Next, note that it follow from (10) and the fact that $1-x-\exp (-x) \leq 0$ for all real $x$ that

$$
\Delta z(j) \leq-q_{1}(j)\left\{\exp \sum_{i=j-\sigma}^{j} b_{\theta}(i)\right\} z(j-\sigma),
$$

for all large $j$, where

$$
z(j)=v(j) \exp \sum_{i=0}^{j-1} b_{\theta}(i)
$$

We summarize these as follows.

Theorem 1. In addition to the conditions imposed on the system (1), suppose further that $0 \leq b_{\theta}(j)=q_{0}(j)-h(j) c(j) \theta$, and either $b_{\theta}(j)<1$ or $q_{1}(j) \geq 0$ for 
all large $j$. If system (1) is $\Psi_{\theta}$-nonoscillatory, then the recurrence relations (11) and (12) have eventually positive solutions.

Theorem 2. In addition to the conditions imposed on the system (1), suppose further that $b_{\theta}(j)=q_{0}(j)-h(j) c(j) \theta$ for all large $j$. If system (1) is $\Psi_{\theta}$-nonoscillatory, then the recurrence relation (12) has an eventually positive solution.

Oscillatory behavior of recurrence relations of the form (11) or (12) has been investigated in a few recent studies. For instance, we have the following result [3, Theorem 7.6.1].

Lemma 2. Assume that $q(n)$ is a nonnegative sequence of real numbers and let $\sigma$ be positive integer. Suppose that

$$
\liminf _{n \rightarrow \infty}\left\{\frac{1}{\sigma} \sum_{i=n-\sigma}^{n-1} q(i)\right\}>\frac{\sigma^{\sigma}}{(\sigma+1)^{\sigma+1}} .
$$

Then the recurrence relation

$$
\Delta x(n)+q(n) x(n-\sigma) \leq 0, \quad n=0,1,2, \ldots
$$

cannot have an eventually positive solution.

Another elementary result is the following.

Lemma 3. Assume that $q(n)$ is a sequence of real numbers such that $1-q(n)$ is not eventually positive, then

$$
\Delta x(n)+q(n) x(n) \leq 0, \quad n=0,1,2, \ldots
$$

cannot have an eventually positive solution.

Proof. For otherwise if $\{x(n)\}$ is an eventually positive solution of the above relation, then

$$
1-q(n) \geq x(n+1) / x(n)
$$

for all large n. Q.E.D.

In case the inequality condition in Lemma 2 cannot be applied, the following may be used.

Lemma 4. Assume that $q(n)$ is a sequence of nonnegative numbers and $\sigma$ is a positive integer. If

$$
\liminf _{n \rightarrow \infty} \sum_{i=n-\sigma}^{n-1} q(i)>\delta>0, \quad \delta<\left\{\frac{\sigma}{\sigma+1}\right\}^{\sigma+1}
$$


and

$$
\limsup _{n \rightarrow \infty} \sum_{i=n-\sigma}^{n} q(i)>1-\frac{\delta^{2}}{2(1-\delta+\sqrt{(1-2 \delta)})}
$$

then (13) cannot have an eventually positive solution.

Proof. Suppose to the contrary that $\{y(n)\}$ is an eventually positive solution of (13), then $\mathrm{n}$ view of (13), $\{y(n)\}$ is an eventually nonincreasing sequence. We assert further that for all large $j$,

$$
y(j+1) \geq y(j-\sigma) / \mu_{1},
$$

where

$$
\mu_{1}=\frac{4}{\delta^{2}}\left\{1-\frac{\delta}{2}\right\}
$$

To see this, note first that in view of (14), if $j$ is large enough, there is an integer $n$ such that $n-\sigma \leq j \leq n$ and

$$
\sum_{i=n-\sigma}^{j} q(i) \geq \frac{\delta}{2}, \quad \sum_{i=j+1}^{n} q(i) \geq \frac{\delta}{2}
$$

Summing (13) from $n-\sigma$ to $j$, and utilizing the fact that $\{y(n)\}$ is eventually nonincreasing, we obtain

$$
y(j+1)-y(n-\sigma)+\frac{\delta}{2} y(j-\sigma) \leq y(j+1)-y(n-\sigma)+\sum_{i=n-\sigma}^{j} q(i) y(i-\sigma) \leq 0 .
$$

Similarly, summing (13) from $j+1$ to $n$, and utilizing the fact that $\{y(n)\}$ is eventually nonincreasing, we obtain

$$
y(n+1)-y(j+1)+\frac{\delta}{2} y(n-\sigma) \leq 0 .
$$

Combining (15) and (16), we obtain

$$
y(j+1) \geq y(n+1)+\frac{\delta}{2}\left\{y(j+1)+\frac{\delta}{2} y(j-\sigma)\right\},
$$

which implies $y(j+1) \geq y(j-\sigma) / \mu_{1}$. Next, we assert that for all large $j$,

$$
y(j+1) \geq y(j-\sigma) / \mu_{2}
$$

where

$$
\mu_{2}=\frac{4(1-\delta)}{\delta^{2}}<\mu_{1}
$$


Indeed, since for all large $n$, we have shown that $y(n+1) \geq y(n-\sigma) / \mu_{1}$, thus by substituting this inequality into (16), we obtain

$$
y(j+1) \geq y(n+1)+\frac{\delta}{2} y(n-\sigma) \geq y(n-\sigma)\left\{\frac{1}{\mu_{1}}+\frac{\delta}{2}\right\} .
$$

In view of $(15)$, we obtain further that

$$
y(j+1) \geq\left(y(j+1)+\frac{\delta}{2} y(j-\sigma)\right)\left\{\frac{1}{\mu_{1}}+\frac{\delta}{2}\right\}
$$

Hence,

$$
\frac{y(j-\sigma)}{y(j+1)} \leq \frac{1-\left\{\frac{1}{\mu_{1}}+\frac{\delta}{2}\right\}}{\frac{\delta}{2}\left\{\frac{1}{\mu_{1}}+\frac{\delta}{2}\right\}}=\frac{4(1-\delta)}{\delta^{2}}=\mu_{2}<\mu_{1},
$$

for all large $j$. In general, we have

$$
y(j+1) \geq y(j-\sigma) / \mu_{m}, \quad m \geq 1
$$

where

$$
\mu_{m}=\frac{1-\left\{\frac{1}{\mu_{m}}+\frac{\delta}{2}\right\}}{\frac{\delta}{2}\left\{\frac{1}{\mu_{m}}+\frac{\delta}{2}\right\}}
$$

By induction, it is not difficult to show that $\left\{\mu_{m}\right\}$ is a positive decreasing sequence which is convergent to some nonnegative limit, say $\mu$. By taking limits on both sides of (17) and then solving for $\mu$, we obtain

$$
\mu=\frac{2}{\delta^{2}}(1-\delta+\sqrt{(1-2 \delta)})
$$

In other words, we have shown that $y(j+1) \geq y(j-\sigma) / \mu$ for all large $j$.

Finally, by summing (13) from $j-\sigma$ to $j$, we obtain

$$
y(j+1)-y(j-\sigma)+\sum_{i=j-\sigma}^{j} q(i) y(j-\sigma)=0 .
$$

By substituting the inequality $y(j+1) \geq y(j-\sigma) / \mu$ into the above equation, we obtain

$$
y(j-\sigma)\left\{\sum_{i=j-\sigma}^{j} q(i)-1+\frac{\delta^{2}}{2(1-\delta+\sqrt{(1-2 \delta)})}\right\} \leq 0
$$

for all large $j$, which is a contradiction. The proof is complete.

In view of these results and others, we may then obtain oscillation criteria for our system (1). For instance, we have shown that if (1) is $\Psi_{\theta}$-nonoscillatory, then (11) has 
an eventually positive solution. In view of Lemma 2, we see that if in addition to the conditions imposed on the system $(1), q_{1}(j)$ is a sequence of nonnegative integers, $\sigma$ is a positive integer, and $b_{\theta}=q_{0}(j)-h(j) c(j) \theta \geq 0$ for all large $j$, then a contradiction will be reached, that is, (1) must be $\Psi_{\theta}$-oscillatory.

Corollary 1 . In addition to the conditions imposed on the system (1), suppose further that $\sigma>0, b_{\theta}(j)=q_{0}(j)-h(j) c(j) \theta \geq 0$, and $q_{1}(j)$ is nonnegative for all large $j$ such that

$$
\liminf _{n \rightarrow \infty}\left\{\frac{1}{\sigma} \sum_{i=n-\sigma}^{n-1} \frac{q_{1}(j)}{1-b_{\theta}(j)}\right\}>\frac{\sigma^{\sigma}}{(\sigma+1)^{\sigma+1}}
$$

then (1) is $\Psi_{\theta}$-oscillatory.

For the sake of convenience, let us denote

$$
q(j)=q_{1}(j)\left\{\exp \sum_{i=j-\sigma}^{j} b_{\theta}(i)\right\}
$$

in the next two corollaries. the follwoing result follows from Lemma 3 and theorem 2.

Corollary 2. In addition to the conditions imposed on the system (1), suppose further that $\sigma=0, b_{\theta}(j)=q_{0}(j)-h(j) c(j) \theta$ for all large $j$, and $1-q(j)$ is not eventually positive, then (1) is $\Psi_{\theta}$-oscillatory.

Similarly, the following result follows from Lemmas 2, 4 and Theorem 2.

Corollary 3. In addition to the conditions imposed on the system (1), suppose further that $\sigma>0, b_{\theta}(j)=q_{0}(j)-h(j) c(j) \theta$ and $q_{1}(j)$ is nonnegative for all large $j$ such that

$$
\liminf _{n \rightarrow \infty} \sum_{j=n-\sigma}^{n-1} q(j)>\delta
$$

where either

$$
\delta \geq \frac{\sigma^{\sigma+1}}{(\sigma+1)^{\sigma+1}}
$$

or

$$
0<\delta<\frac{\sigma^{\sigma+1}}{(\sigma+1)^{\sigma+1}} \quad \text { and } \quad \limsup _{n \rightarrow \infty} \sum_{j=n-\sigma}^{n} q(j)>1-\frac{\delta^{2}}{2(1-\delta+\sqrt{(1-2 \delta)}}
$$

then (1) is $\Psi_{\theta}$-oscillatory.

Further combinations of Theorems 1 and 2 and Lemmas 2, 3 and 4 are possible. The principle, however, is the same. 
We can make use of (8) and derive another oscillation criteria for (1). This criterion does not need the assumption that $b_{\theta}(j) \geq 0$.

Theorem 3. In addition to the conditions imposed on system (1), assume further that

$$
\liminf _{j \rightarrow \infty} q_{1}(j)>\frac{B^{\sigma+1} \sigma^{\sigma}}{(\sigma+1)^{\sigma+1}}
$$

where $B=\limsup _{j \rightarrow \infty}\left(1-b_{\theta}(j)\right)<\infty$. Then every solution of $(1)$ is oscillatory.

Proof. Suppose not, then we have already shown that

$$
v(j+1)-\left(1-b_{\theta}(j)\right) v(j)+q_{1}(j) v(j-\sigma) \leq 0
$$

has an eventually positive solution $v(j)$. Dividing the above relation by $v(j)$, we obtain

$$
\frac{v(j+1)}{v(j)}-\left(1-b_{\theta}(j)\right)+q_{1}(j) \frac{v(j-\sigma)}{v(j)} \leq 0
$$

Set $r_{j}=v(j) / v(j+1)>0$, then

$$
r_{j}^{-1} \leq 1-b_{\theta}(j)-q_{1}(j) r_{j-\sigma} r_{j-\sigma+1} \cdots r_{j-1} .
$$

Set $\liminf \inf _{j \rightarrow \infty} r_{j}=R$. Then $R \geq 0$. $R$ cannot be zero for otherwise we can multiply (18) through by $r_{j}$ and then obtain

$$
1 \leq \liminf _{j \rightarrow \infty}\left(r_{j}-b_{\theta}(j) r_{j}-q_{1}(j) r_{j-\sigma} r_{j-\sigma+1} \cdots r_{j-1} r_{j}\right)=0,
$$

which is a contradiction. From (18), we then have

$$
\limsup _{j \rightarrow \infty} \frac{1}{r_{j}}=\frac{1}{R} \leq B-R^{\sigma} \liminf _{j \rightarrow \infty} q_{1}(j)
$$

This implies

$$
\liminf _{j \rightarrow \infty} q_{1}(j) \leq \frac{B-R^{-1}}{R^{\sigma}} \leq \max _{R>0} \frac{B-R^{-1}}{R^{\sigma}}=\frac{B^{\sigma+1} \sigma^{\sigma}}{(\sigma+1)^{\sigma+1}}
$$

which is a contradiction. The proof is complete.

2. In this section, we consider a more general direct control system of the form

$$
\Delta_{2}\left\{u(i, j)-\sum_{k=1}^{m} d_{k}(j) u\left(i, j-\tau_{k}\right)\right\}=a_{0}(j) \Delta_{1}^{2} u(i-1, j)+\sum_{k=1}^{s} a_{k}(j) \Delta_{1}^{2} u\left(i-1, j-\rho_{k}\right)
$$




$$
-q_{0}(i, j) u(i, j)-\sum_{k=1}^{p} q_{k}(i, j) u\left(i, j-\sigma_{k}\right)+h(j) f(c(j) u(i, j)), \quad 1 \leq i \leq n, j \geq 0,
$$

where $m, s, p$ are positive integers, the delays $\tau_{1}, \cdots, \tau_{m}, \rho_{1}, \cdots, \rho_{s}, \quad \sigma_{1}, \cdots, \sigma_{p}$ are nonnegative integers, the functions $d_{0}(j), \ldots, d_{m}(j), q_{0}(i, j), \ldots, q_{p}(i, j), a_{0}(j), \ldots, a_{s}(j)$, $h(j), c(j)$ are nonnegative for $1 \leq i \leq n$ and/or $j \geq 0$, and $f$ belongs to the angular domain $\Psi_{\theta}$ defined by (2). This system can be regard as a discrete analog of a parabolic equation of the neutral type [2, Chapter 6],

$$
\begin{aligned}
\frac{\partial}{\partial t}\left\{u-\sum_{k=1}^{m} d_{k}(t) u\left(x, t-\tau_{k}\right)\right\}= & a_{0}(t) u_{x x}+\sum_{k=1}^{s} a_{k}(t) u_{x x}\left(x, t-\rho_{k}\right) \\
& -q_{0}(x, t) u-\sum_{k=1}^{p} q_{k}(x, t) u\left(x, t-\sigma_{k}\right)+h(t) f(c(t) u) .
\end{aligned}
$$

Let $\tau=\max \left\{\tau_{1}, \ldots, \tau_{m}, \rho_{1}, \ldots, \rho_{s}, \sigma_{1}, \ldots, \sigma_{p}\right\}$. Then under the conditions

$$
\begin{gathered}
u(0, j)=0=u(n+1, j), \quad j \geq 0 \\
u(i, j)=\varphi(i, j), \quad-\tau \leq j \leq 0,0 \leq i \leq n+1
\end{gathered}
$$

where $\varphi$ is a given function for $-\tau \leq j \leq 0$ and $0 \leq i \leq n+1$ satisfying $\varphi(0,0)=0=$ $\varphi(n+1,0)$, a unique solution $u(i, j ; \varphi)$ exists. Given an angular domain $\Psi_{\theta}$, suppose there is a function $f$ in $\Psi_{\theta}$ such that a solution $u(i, j)$ of $(19)$ satisfies $u(i, j)>0$ for $1 \leq i \leq n$ and $j \geq T$, or $u(i, j)<0$ for $1 \leq i \leq n$ and $j \geq T$, then letting $\delta=\operatorname{sign} u(i, j)$ and $w(i, j)=\delta u(i, j)$, we see that $w(i, j)>0$ for $1 \leq i \leq n$ and $j \geq T$, and $w\left(i, j-\tau_{k}\right)>$ $0, w\left(i, j-\rho_{k}\right)>0, w\left(i, j-\sigma_{k}\right)>0$ for $1 \leq i \leq n$ and $j \geq T+\tau$. If we let

$$
\begin{gathered}
Q_{k}(j)=\max \left\{q_{k}(i, j) \mid 1 \leq i \leq n\right\}, \quad 0 \leq k \leq p \\
b_{\theta}(j)=Q_{0}(j)-h(j) c(j) \theta
\end{gathered}
$$

and

$$
G(j)=\sum_{t, i=1}^{n} g(t, i) w(i, j)
$$

then $G(j)$ is eventually positive and it is not difficult to follow the same arguments as explained in the last section and arrive at the following recurrence relation

$$
\Delta\left\{G(j)-\sum_{k=1}^{m} d_{k}(j) G\left(j-\tau_{k}\right)\right\} \leq-b_{\theta}(j) G(j)-\sum_{k=1}^{p} Q_{k}(j) G\left(j-\sigma_{k}\right)
$$

for all large $j$.

We now assume that at least one of the functions $Q_{k}(j)$ is eventually bounded below by a positive constant, that the functions $d_{k}(j)(1 \leq k \leq m)$ are eventually bounded 
above by constants $D_{k}(1 \leq k \leq m)$ and that $D_{1}+\cdots+D_{m} \leq 1$. We assert that the function

$$
H(j)=G(j)-\sum_{k=1}^{m} d_{k}(j) G\left(j-\gamma_{k}\right)
$$

is eventually positive if $b_{\theta}(j) \geq 0$. Indeed, we note first that $\Delta H(j)<0$ for $j$ larger than or equal to some $T$. If $H(J) \leq 0$ for some $J \geq T$, then

$$
0>H(J+1)=\sup _{j>J} H(j) \geq \sup _{j>J} G(j)-\sum_{k=1}^{m} D_{k} \sup _{j>J} G(j)=\sup _{j>J} G(j)\left\{1-\sum_{k=1}^{m} D_{k}\right\} \geq 0,
$$

which is a contradiction.

Next note that $H(j) \leq G(j)$ for all large $j$. Thus assuming $b_{\theta}(j) \geq 0$, we see from (23) that

$$
\Delta H(j) \leq-b_{\theta}(j) H(j)-\sum_{k=1}^{p} Q_{k}(j) H\left(j-\sigma_{k}\right)
$$

for all large $j$.

The recurrence relation (24) is more general in form than the relation (8). However, we can modify the arguments used in the last section to obtain recurrence relations of the form (11) and (12). To see this, we first note that $b_{\theta}(j)<1$ for all large $j$, as can be seen from (24) and

$$
\left(1-b_{\theta}(j)\right) H(j) \geq H(j+1)+\sum_{k=1}^{p} Q_{k}(j) H\left(j-\sigma_{k}\right) \geq 0 .
$$

Next, if we rewrite (24) as

$$
\left(1-b_{\theta}(j)\right) \Delta H(j) \leq-b_{\theta}(j) H(j+1)-\sum_{k=1}^{p} Q_{k}(j) H\left(j-\sigma_{k}\right) .
$$

Then assuming $0 \leq b_{\theta}(j)$ for all large $j$, we have

$$
\Delta H(j) \leq-\sum_{k=1}^{p} \frac{Q_{k}(j)}{1-b_{\theta}(j)} H\left(j-\sigma_{k}\right)
$$

for all large $j$. Next, let $\sigma_{*}=\min \left\{\sigma_{1}, \ldots, \sigma_{p}\right\}$. Then since $\Delta H(j) \leq 0$ for all large $j$, $-H\left(j-\sigma_{k}\right) \leq-H\left(j-\sigma_{*}\right)$ for $1 \leq k \leq p$ and all large $j$. This implies

$$
\Delta H(j) \leq-\sum_{k=1}^{p} \frac{Q_{k}(j)}{1-b_{\theta}(j)} H\left(j-\sigma_{*}\right)
$$

for all large $j$. 
Similarly, if we follow the same arguments used to obtain (12), we shall arrive at

$$
\Delta U(j) \leq-\sum_{k=1}^{p} Q_{k}(j) \exp \left\{\sum_{i=j-\sigma_{k}}^{j} b_{\theta}(i)\right\} U\left(j-\sigma_{k}\right)
$$

and then

$$
\Delta U(j) \leq-\sum_{k=1}^{p} Q_{k}(j) \exp \left\{\sum_{i=j-\sigma_{k}}^{j} b_{\theta}(i)\right\} U\left(j-\sigma_{*}\right)
$$

for all large $j$, where

$$
U(j)=H(j) \exp \left\{\sum_{i=0}^{j-1} b_{\theta}(i)\right\} .
$$

We summarize the above derivations as follows.

Theorem 4. In addition to the conditions imposed on system (19), suppose further that $(i) Q_{k}(j)(1 \leq k \leq p)$ and $b_{\theta}(j)$ are defined by (20) and (21) respectively; (ii) $0 \leq b_{\theta}(j)$ for all large $j$; (iii) $d_{k}(j)(1 \leq k \leq m)$ are eventually bounded above by constants $D_{k}(1 \leq k \leq m)$ and $D_{1}+\cdots+D_{m} \leq 1$, and (iv) at least one $Q_{k}(j)$ is eventually bounded below by a positive positive constant. If (19) is $\Psi_{\theta}$-nonoscillatory, then the recurrence relations (26), (27), (28) and (29) have eventually positive solutions.

In view of Lemmas 2, 3, 4 and Theorem 1, we may derive several oscillation criteria. Two examples are given below.

Corollary 4. Under the assumptions of Theorem 4, suppose further that $\rho=\min \left\{\sigma_{1}, \ldots, \sigma_{m}\right\}>0$, and

$$
\liminf _{n \rightarrow \infty}\left\{\sum_{j=n-\rho}^{n-1} \sum_{k=1}^{p} \frac{Q_{k}(j)}{1-b_{\theta}(j)}\right\}>\delta
$$

whrer either

$$
\delta \geq \frac{\rho^{\rho+1}}{(\rho+1)^{\rho+1}}
$$

or,

$$
0<\delta<\frac{\rho^{\rho+1}}{(\rho+1)^{\rho+1}} \text { and } \limsup _{n \rightarrow \infty}\left\{\sum_{j=n-\rho}^{n-1} \sum_{k=1}^{p} \frac{Q_{k}(j)}{1-b_{\theta}(j)}\right\}>1-\frac{\delta^{2}}{2(1-\delta+\sqrt{(1-2 \delta)}}
$$

then system (19) is $\Psi_{\theta}$-oscillatory. 
Corollary 5. Under the assumptions of Theorem 4, if $\sigma_{k}=0$ for $1 \leq k \leq p$ and

$$
1-\frac{1}{1-b_{\theta}(j)} \sum_{k=1}^{p} Q_{k}(j)
$$

is not eventually positive, then system (19) is $\Psi_{\theta}$-oscillatory.

Other combinations of Theorem 2 and Lemmas 2, 3 and 4 are possible, no new principle is involved, however.

3. In this section, we consider a direct control system fo the form

$$
\begin{gathered}
\Delta_{2}^{2} u(i, j-1)=a_{0}(j) \Delta_{1}^{2} u(i-1, j)+\sum_{k=1}^{s} a_{k}(j) \Delta_{1}^{2} u\left(i-1, j-\rho_{k}\right) \\
-q_{0}(i, j) u(i, j)-\sum_{k=1}^{P} Q_{k}(i, j) u\left(i, j-\sigma_{k}\right)+h(j) f(c(j) u(i, j)), 1 \leq i \leq n, j \geq 0,
\end{gathered}
$$

where $s, p$ are positive integers, the delays $\rho_{1}, \cdots, \rho_{s}, \sigma_{1}, \cdots, \sigma_{p}$ are nonnegative integers, the functions $a_{0}(j), \cdots, a_{s}(j), q_{0}(i, j), \cdots, q_{p}(i, j), h(j), c(j)$ are nonnegative for $1 \leq i \leq$ $n$ and/or $j \geq 0$, and $f$ belongs to the angular domain $\Psi_{\theta}$ defined by (2). This system can be regarded as a discrete analog of a hyperbolic equation with delays [2, Chapter 6],

$$
\begin{aligned}
\frac{\partial^{2} u}{\partial t^{2}}= & a_{0}(t) u_{x x}+\sum_{k=1}^{s} a_{k}(t) u_{x x}\left(x, t-\rho_{k}\right)-q_{0}(x, t) u \\
& -\sum_{k=1}^{p} q_{k}(x, t) u\left(x, t-\sigma_{k}\right)+h(t) f(c(t) u) .
\end{aligned}
$$

Let $\tau=\max \left\{1, \rho_{1}, \cdots, \rho_{s}, \sigma_{1}, \cdots, \sigma_{p}\right\}$. Then under the conditions

$$
\begin{gathered}
u(0, j)=0=u(n+1, j), \quad j \geq 0 \\
u(i, j)=\varphi(i, j), \quad-\tau \leq j \leq 0,0 \leq i \leq n+1,
\end{gathered}
$$

where $\varphi(i, j)$ is a given function for $-\tau \leq j \leq 0$ and $0 \leq i \leq n+1$ satisfying $\varphi(0,0)=$ $\varphi(n+1,0)$, a unique solution $u(i, j ; \varphi)$ exists. Given an angular domain $\Psi_{\theta}$, suppose there is a function $f$ in $\Psi_{\theta}$ such that a solution $u(i, j)$ of $(30)$ satisfies $u(i, j)>$ 0 for $1 \leq i \leq n$ and $j \geq T$, or $u(i, j)<0$ for $1 \leq i \leq n$ and $j \geq T$, then letting $\delta=\operatorname{sign} u(i, j)$ and $w(i, j)=\delta u(i, j)$, we see that $w(i, j)>0$ for $1 \leq i \leq n$ and $j \geq T$, and $w\left(i, j-\rho_{k}\right)>0$ and $w\left(i, j-\sigma_{k}\right)>0$ for $1 \leq i \leq n$ and $j \geq T+\tau$. If we let $Q_{k}(j)$ and $b_{\theta}(j)$ be respectively defined by (20) and (21), and let

$$
V(j)=\sum_{t, i=1}^{n} g(t, i) w(i, j)
$$


then $V(j)$ is eventually positive and it is not difficult to follow the same arguments as explained in the first section and arrive at the follow recurrence relation

$$
\Delta^{2} V(j-1) \leq-b_{\theta}(j) V(j)-\sum_{k=1}^{p} Q_{k}(j) V\left(j-\sigma_{k}\right)
$$

for all large $j$.

Assuming the $b_{\theta}(j) \geq 0$ for all large $j$, we see from (31) that $\Delta^{2} V(j) \leq 0$ for all large $j$. Since a concave, positive sequence is eventually nondecreasing, $\Delta V(j) \geq 0$ for all large $j$. This implies

$$
V(j)=\Delta V(j-1)+V(j-1) \geq \Delta V(j-1)
$$

for all large $j$. Therefore, from (31), we see that

$$
\Delta^{2} V(j-1) \leq-b_{\theta}(j) \Delta V(j-1)-\sum_{k=1}^{p} Q_{k}(j) \Delta V\left(j-1-\sigma_{k}\right)
$$

Thus

$$
\Delta Y(j) \leq-b_{\theta}(j+1) Y(j)-\sum_{k=1}^{p} Q_{k}(j+1) Y\left(j-\sigma_{k}\right)
$$

for all large $j$, where $Y(j)=\Delta V(j)$ (which is eventually positive).

Note that the recurrence relation (33 is similar to the relation (24). Thus by means of resoning similar to that used to derive Theorem 1 , we have the following result.

Theorem 6. In addition to the conditions imposed on system (30), suppose further that (i) $Q_{k}(j)(1 \leq k \leq p)$ and $b_{\theta}(j)$ are defined by (20) and (21) respectively; (ii) $0 \leq b_{\theta}(j)$ for all large $j$, and (iii) $\sigma_{*}=\min \left(\sigma_{1}, \cdots, \sigma_{p}\right)$. If (30) is $\Psi_{\theta}$-nonoscillatory, then the following recurrence relations

$$
\begin{aligned}
& \Delta X(j) \leq-\sum_{k=1}^{p} \frac{Q_{k}(j+1)}{1-b_{\theta}(j+1)} X\left(j-\sigma_{k}\right) \\
& \Delta X(j) \leq-\sum_{k=1}^{p} \frac{Q_{k}(j+1)}{1-b_{\theta}(j+1)} X\left(j-\sigma_{*}\right) \\
& \Delta X(j) \leq-\sum_{k=1}^{p} Q_{k}(j+1) \exp \left\{\sum_{i=j-\sigma_{k}}^{j} b_{\theta}(i+1)\right\} X\left(j-\sigma_{k}\right)
\end{aligned}
$$

and

$$
\Delta X(j) \leq-\sum_{k=1}^{p} Q_{k}(j+1) \exp \left\{\sum_{i=j-\sigma_{k}}^{j} b_{\theta}(i+1)\right\} X\left(j-\sigma_{*}\right)
$$


have eventually positive solutions.

In view of this Theorem and Lemmas 2,3 and 4, we can obtain oscillation criteria similar to the Corollaries mentioned in Sections 1 and 2. The details are omitted because no new principle is involved.

\section{Acknowledgement}

The first author is partially supported by the National Science Council of R.O.C. and the third author is partially supported by NNSF of China.

\section{References}

[1] S. S. Cheng, "A discrete analogue of the inequality of Lyapunov," Hokkaido Math. J., 12, $105-112$.

[2] D. D. Bainov and D. P. Mishev, Oscillation Theory for Neutral Differential Equatins with Delay, Adam Hilger, 1991.

[3] I. Gyori and G. Ladas, Oscillation Theory of Delay Differential Equations, Clarendon Press, Oxford, 1991.

[4] S. S. Cheng and B. G. Zhang, "Qualitative theory of partial difference equations (I): Oscillation of nonlinear partial difference equations," Tamkang J. Math., 25 (1994), 279-288.

Department of Mathematics, Tsing Hau University, Hsinchu, Taiwan, 30043.

Department of Mathematics, Jinzhou Teacher's College, Hubei, China 434100.

Department of Applied Mathematics, Ocean University of Qingdao, China, 266003 